Dies soll sich jetzt ändern: die allgemeine Bildung soll hinsichtlich aller Merkmale vor Diskriminierung geschützt sein Ausnahme: das Geschlecht. Im Juli 2008 hat die Kommission den Vorschlag für eine Richtlinie des Rates zur Anwendung des Grundsatzes der Gleichbehandlung ungeachtet der Religion oder der Weltanschauung, einer Behinderung, des Alters oder der sexuellen Ausrichtung vorgelegt. ${ }^{1}$ Mit dem Erlass einer vierten auf Art. 13 gestützten Richtlinie würde das Mindestschutzniveau für alle in Art. 13 genannten Merkmale bis auf das Geschlecht angeglichen werden. Zum Geschlecht würde die Lücke des Diskriminierungsschutzes für die Sozialleistungen und die allgemeine Bildung zum Geschlecht weiterhin bestehen bleiben. Die vorgeschlagenen Änderungen zeigt Tabelle 2.

Im europäischen Jahr der Chancengleichheit 2007 sind von der EG-Kommission zunächst interessierte Kreise wie die Geschäftswelt, die Sozialpartner sowie - breiter angelegt mit einer Online-Konsultation - auch die Öffentlichkeit zu möglichen Vorschlägen befragt worden.

Der Vorschlag der Kommission entspricht auch einem vom Europäischen Parlament geäußerten Wunsch. ${ }^{2}$ Dort und im Rat wird der Richtlinienentwurf derzeit beraten. ${ }^{3}$

Die Regelungslücke hinsichtlich der Sozialleistungen und zur allgemeinen Bildung würde dem Grundsatz des Art. 3 Abs. 2 EG widersprechen, bei allen Tätigkeiten der Gemeinschaft darauf hinzuwirken, Ungleichheiten zu beseitigen und die
Gleichstellung von Frauen und Männern zu fördern, der ebenfalls seit dem Vertrag von Amsterdam gilt. Zum Tragen kommen die zum Gleichbehandlungsrecht getrennten Diskurse der beteiligten Nichtregierungsorganisationen. So spiegeln sich auch u.a. die für die Bekämpfung von Diskriminierung und der Gleichstellung der Geschlechter getrennten Zuständigkeiten bei der EG-Kommission und beim Europäischen Parlament zwischen dem Ausschuss für Bürgerliche Freiheiten, Justiz und Inneres (LIBE) und für Rechte der Frau und Gleichstellung der Geschlechter (FEMM) mit offensichtlich unterschiedlichen Durchsetzungschancen wider. Anfang 2009 hat der zuständige Ausschuss des Europäischen Parlaments gegenüber der Kommission die Erwartung geäußert, den Schutz gegen Diskriminierung wegen des Geschlechts auf das Niveau zu bringen, das für die anderen in Art. 13 EG genannten Merkmale gilt. Die bestehende Hierarchie ist zu beseitigen. Hierzu werden Vorschläge der Kommission für 2010 erwartet. $^{4}$

\title{
Umsetzung des AGG in den Betrieben: Außer Spesen nichts gewesen?
}

Das Allgemeine Gleichbehandlungsgesetz (AGG) ist seit 14 . August 2006 in Kraft, also seit gut zweieinhalb Jahren. Unsere Unternehmensbefragung wollte untersuchen, ob und wie das AGG nach zwei Jahren umgesetzt wurde und welche Veränderungen es für die betriebliche Praxis gebracht hat. Zwischen Januar und Mai 2008 führte das Hamburger AGG-Projekt ${ }^{1}$ im Großraum Hamburg 52 leitfadengestützte Experteninterviews mit einer Dauer zwischen 40 Minuten und zwei Stunden. Persönlich befragt wurden 52 Personen aus 41 Unternehmen der Privatwirtschaft: 36 Vertreter/innen aus den Personalabteilungen bzw. der Geschäftsleitung, acht Mitglieder des Betriebsrats und acht Beauftragte für Gleichstellung, für Behinderte oder sonstige Minoritäten. Die Befragung war nicht repräsentativ angelegt, verteilte sich aber recht gleichmäßig auf die unterschiedlichen Unternehmenstypen und Branchen. ${ }^{2}$ Ihre Ergebnisse sind damit zumindest für städtische Ballungsräume durchaus aussagekräftig.

\section{Die Beurteilung des AGG bei seiner Einführung}

Die deutsche Wirtschaft lehnte die neuen rechtlichen Vorgaben 2006 mehrheitlich ab und prophezeite Klagewellen von abgelehnten Bewerber/innen und hohe Umsetzungskosten. In einem „Stimmungsbild“ unter Personalmanagern $2006^{3}$ fanden sich Aussagen wie: „Das AGG ist ein Hammergesetz, das uns in vielen Punkten fordern wird.“ „Das AGG lädt dazu ein, es schamlos auszunutzen.“ „Vor allem in der Anfangszeit wird es Klagen hageln.“ „Alles in allem sehen wir zu viel Regelungsund Überwachungsbedarf.“

1 Projektleitung: Sibylle Raasch und Daniela Rastetter, Projektmitarbeiterinnen: Nina Bielau, Diana Fazari, Ursula Kisse, Katarzyna Najlepsza, Emma Patrignani, Tinka Rieckhoff, Regine Starck, Guanayu Hou.

2 Vgl. ausführlicher Ergebnisbericht Raasch, S.; Rastetter, D.: Die Anwendung des AGG in der betrieblichen Praxis, Hamburg Januar 2009.

3 Personalmagazin, Titelthema: Das AGG im Urteil der Personalmanager, 7/2006, S. 28-29.

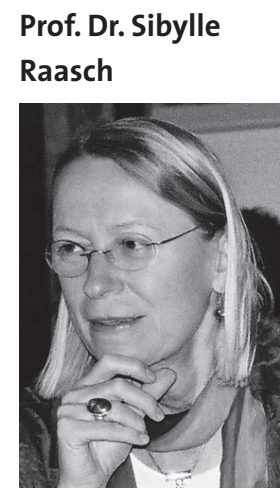

Vorsitzende der Kommission Arbeits-, Gleichstellungs- und Wirtschaftsrecht des djb, Professur für Öffentliches Recht und Gender Studies, Fachbereich Sozialökonomie, Universität Hamburg 


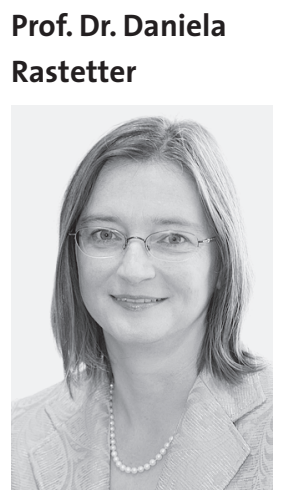

Professur für Personal, Organisation und Gender Studies, Fachbereich Sozialökonomie, Universität Hamburg
Studien aus dem Jahr 2007, also kurz nach der Einführung des AGG, waren zur Überprüfung solcher Vorbehalte wegen ihres frühen Zeitpunktes noch wenig aussagekräftig. Sie kamen zudem zu unterschiedlichen Ergebnissen: Eine Randstad-Befragung von 302 Unternehmen aus dem August $2007^{4}$ zeigte, dass zwar fast alle Befragten das AGG mehr oder weniger gut kannten, das Gesetz also in den Unternehmen angekommen war. Fast drei Viertel der Befragten äußerten sich jedoch neutral bzw. hatten noch keinerlei Erfahrungen mit dem Gesetz. Beschwerden aufgrund des AGG gab es nur bei knapp acht Prozent, zumeist wenige. Hoffjan/Bramann $^{5}$ rechneten die Ergebnisse ihrer Befragung von 501 Unternehmen im Sommer 2007, erstellt im Auftrag der Initiative Neue Soziale Marktwirtschaft, auf gesetzesinitiierte Mehrkosten von 1,73 Milliarden Euro hoch, wovon ca. 40 Prozent fortlaufende Kosten seien. Das AGG müsse deswegen dringend nachgebessert werden. Diese Hochrechnung löste neue öffentliche Diskussionen aus und wurde von der Antidiskriminierungsstelle des Bundes (ADS) wegen ihrer Berechnungsmethode und Schlussfolgerungen als einseitig und nicht valide kritisiert. ${ }^{6}$ Andere Befragungen kommen zu wesentlich geringeren Mehrbelastungen der Betriebe. ${ }^{7}$ 2008 räumte nun selbst das Institut der deutschen Wirtschaft (IWD) ein, dass es, wie schon vor dem AGG, wenig Klagen wegen Diskriminierung gegeben habe und die prognostizierte Klagewelle ausgeblieben sei. ${ }^{8}$ Die juristische Datenbank JURIS habe bis August 2008 nur 251 Klagen zum Stichwort „Normen AGG“ verzeichnet. Die Personalchefs hätten sich offenbar auf das AGG gut vorbereitet, was die Unternehmen allerdings die oben geschätzten 1,7 Mrd. Euro gekostet habe. Unklar bleibt in den bisherigen Studien, was die betrieblichen Akteure tatsächlich getan haben und was sie vom AGG inzwischen halten.

\section{Die Umsetzung des AGG bis Mitte 2008}

\section{Betriebliche Informationspolitik, Schulungen und Beschwerdestellen}

Das AGG will über Arbeitgeberpflichten sicherstellen, dass im Betrieb ein Informationsminimum zum neuen Gesetz hergestellt wird: Das Gesetz ist im Betrieb durch Aushang, Auslegung oder im Betrieb übliche Kommunikationstechnik bekannt zu machen. Weiter sollen die Arbeitgeber ihre Beschäftigten „in geeigneter Art und Weise“ auf die Unzulässigkeit von Diskriminierungen hinweisen, insbesondere im Rahmen der beruflichen Aus- und Fortbildung ( $\mathbb{S} 12$ Abs. 2 Satz 1 AGG). Im Betrieb sind zuständige Beschwerdestellen zu benennen $(\mathbb{S} 12$
Abs. 5 AGG). Eine besondere Beschwerdestelle, wie Anfangs noch im Gesetzesentwurf vorgesehen, muss hingegen nicht eingesetzt werden.

Nach unseren Befragungsergebnissen sind die Unternehmen ihrer Informationspflicht weitgehend nachgekommen. 83 Prozent haben ihre Beschäftigten über das Gesetz informiert. Häufigstes Informationsmedium war mit 46 Prozent das Rundschreiben in Papierform oder via Intranet. Eine beachtliche Minderheit von 13 Prozent der Unternehmen - fast ausschließlich kleine und mittlere Unternehmen (KMU) - vernachlässigte ihre Informationspflichten allerdings gänzlich.

Schulungen zum AGG führten nur 71 Prozent der Betriebe durch, jedoch häufig nur selektiv für Führungskräfte und/oder Personalverantwortliche, obwohl das Gesetz Schulungen des Arbeitgebers für „seine Beschäftigten“ fordert ( $\$ 12$ Abs. 2 Satz 2 AGG). Eine Entlassung aus der Haftung nach SS 15 Abs. 1, 12 Abs. 2 Satz 2 AGG tritt jedenfalls nur ein, wenn die Beschäftigten, die diskriminiert haben, zuvor vom Arbeitgeber geschult worden sind. ${ }^{9}$ Das scheint vielen Unternehmen nicht klar zu sein. Da Diskriminierungen nicht nur im Bereich der Personalverwaltung bei Einstellungen, Beförderungen oder dem Entgelt vorkommen, sondern auch durch die Arbeitsbedingungen erfolgen können, insbesondere durch Belästigung oder sexuelle Belästigung, sollte eine breitere Schulung auch im Interesse der Arbeitgeber liegen. Andererseits sind Geschulte nicht nur (verhinderte) Diskriminierer/ innen, sondern vielleicht auch künftige Kläger/ innen wegen Diskriminierung. Das Interesse der Unternehmen an Schulungen ist also zwiespältig, auch unabhängig von den durch die Schulungen entstehenden Kosten. Geschult wurde zu 48 Prozent intern und zu 29 Prozent extern, teilweise auf beiden Wegen. Insbesondere Arbeitgeberverbände

4 Randstad-Expertenpanel Personal: Das Allgemeine Gleichbehandlungsgesetz (AGG). Ergebnisse der Umfragewelle August 2007, 10.9.2007.

5 Hoffjan, A.; Bramann, A.: Gesetzesfolgekosten des Allgemeinen Gleichbehandlungsgesetzes (AGG). Betriebs-Berater, 2007, S. 2625-2629.

6 ADS: Pressemitteilung Nr. 7 vom 14.8.2008; Bundesregierung: Kosten des Allgemeinen Gleichbehandlungsgesetzes. Siehe auch Antwort der Bundesregierung auf die Kleine Anfrage der Abgeordneten Rainer Brüderle u.a. und der Fraktion der FDP, BT Drs. 16/10728 vom 30.10.2008.

7 Deutsches Institut für kleine und mittlere Unternehmen e.V.: Das Allgemeine Gleichbehandlungsgesetz in KMU - Erfahrungen, Erwartungen, Maßnahmen und Meinungen. Berlin 2007.

8 IWD: Kaum Klagen wegen Diskriminierung, in: iwd 11.12.2008, S. 8.

9 Vgl. Bauer, J.-H.; Göpfert, B.; Krieger, St.: Allgemeines Gleichbehandlungsgesetz. 2. Auflage, München 2008, $\S 12 \mathrm{Rn} .27$. 
und Gewerkschaften wurden als Träger externer Schulungen genannt. Rechtsanwälte/innen und Berater/innen kamen nur selten zum Zuge.

Als vorbildlich können zwei Großunternehmen bezeichnet werden, die als Schulung u.a. einen internen Test für alle Beschäftigten angeboten haben. Kleinunternehmen haben eher seltener geschult. Manchmal wurde sogar eine rigide Ablehnung jeder Art von Schulung durch die Geschäftsleitung bekundet: „Nein, wir haben gar nichts gemacht. Wir haben jedem freigestellt, dass er die Bild-Zeitung liest, da stand alles drin ... Da habe ich keinen Handlungsbedarf gesehen. " (Geschäftsführer, Großunternehmen, Private Dienstleistungen).

Neu eingerichtet wurde nirgends eine Beschwerdestelle. Aber ebenfalls 71 Prozent der Betriebe haben zumindest eine AGG-Beschwerdestelle benannt. Bei 23 Prozent war dies nicht der Fall. Teilweise wurde auch hier deutliche Ablehnung geäußert: „Der Firmeninhaber und der liebe Gott (entscheiden, die Verf.). Da braucht man keine andere Beschwerdestelle. “ (Geschäftsführer, Großunternehmen, private Dienstleistungen). Wo vorhanden, ist die Beschwerdestelle in mehr als der Hälfte der Fälle (57 Prozent) in der Personalabteilung oder gar bei der Unternehmensleitung selbst angesiedelt. Das sind aber Stellen, an die sich Beschäftigte in solchen Fällen als erste Anlaufstelle eher ungern wenden dürften. So sahen das auch einige befragte Personalverantwortliche selbst.

Bei einigen Großunternehmen wurden Personalstelle, Betriebsrat und gegebenenfalls noch Gleichstellungsbeauftragte nebeneinander als Beschwerdestellen benannt. Womit sichergestellt sein dürfte, dass jede/r Hilfesuchende hier eine passende Ansprechstelle findet. Vor allem kleinere Betriebe ignorieren jedoch die gesetzliche Verpflichtung, eine geeignete Beschwerdestelle zu benennen. Dabei dürfte es Benachteiligten in kleineren Betrieben sowieso besonders schwer fallen sich zu beschweren, besonders wenn es um ein Verhalten der Chefin oder des Chefs persönlich geht. Eine externe Beschwerdestelle, zum Beispiel bei der zuständigen Kammer, konnten sich dennoch auf Nachfrage nur drei Kleinunternehmen vorstellen, ebenso viele lehnten diese Lösung ab.

\section{Änderungen in der Einstellungspraxis und bei der Personal- entwicklung}

Knapp 80 Prozent (41) der Befragten sagten, dass es wegen des AGG Änderungen im Einstellungsverfahren gegeben habe. $37 \mathrm{Mal}$ wurden die Ausschreibungstexte überprüft und gegebenenfalls angepasst. Die Formulierung ,jung und dynamisch" wurde 13 Mal und geschlechtsspezifische Formulierungen wurden sieben Mal nicht mehr verwendet. Zwei Unternehmen haben jetzt sicherheitshalber einen externen Berater mit der Stellenausschreibung beauftragt. Die Einstellungsgespräche werden häufiger von zwei Personen geführt und öfter werden Protokolle erstellt.

Bei den Bewerbungsunterlagen greift man gerne zur Formulierung „bitte senden Sie die üblichen Unterlagen“. Damit vermeidet man die Forderung nach einem Bewerbungsfoto, die nach dem AGG problematisch sein könnte, schließt das
Zusenden eines Fotos aber auch nicht aus. Einige Personalverantwortliche geben offen zu, dass sie hoffen, die Bewerber würden weiterhin ein Foto schicken.

Bei der Dokumentation der Unterlagen, die im Rahmen des AGG einen hohen Beweiswert hat, hat die eine Hälfte der Betriebe wegen des AGG den Aufwand erhöht, die andere Hälfte verfährt wie vor dem Inkrafttreten des Gesetzes. Die Personalreferentin eines Großunternehmens sagt dazu: „Wenn ich da ganz sauber arbeiten wollte, müsste ich das dokumentieren, das wäre ein erheblicher Personalaufwand. Und da kann man die Rechnung stellen: Wenn sich jemand einklagt und der bekommt höchstens drei Monatsgehälter, dann ist das sicherlich am Ende weniger als wenn wir den Personalaufwand nach oben treiben. Wir haben 10000 Bewerber, von denen werden am Ende 100 eingestellt, das würde ins Unermessliche gehen.“ Ein Beleg dafür, dass in der Praxis abgewogen wird zwischen Kosten, die durch das Gesetz, und Kosten, die durch einen Gesetzesbruch entstehen. Wobei die Kosten eines Gesetzesbruchs eher geringer veranschlagt werden.

Bei den Einstellungsinterviews gehen die meisten Betriebe wie bisher vor, einige führen die Gespräche aufgrund des AGG jetzt zu zweit. Nur selten wurden einige Fragen im Interview modifiziert. Kein Unternehmen hat die eigentlichen betriebsspezifischen Einstellungskriterien geändert.

Insgesamt entsteht der Eindruck, dass im Rekrutierungsprozess jene Verfahrenselemente überprüft wurden, bei denen die Gefahr bestand, dass ein Verstoß gegen das AGG leicht erkennbar würde. Die Betriebe versuchen, ihre bisherige Praxis beizubehalten, ohne mit dem Gesetz erkennbar in Konflikt zu geraten. Stattdessen wird von vielen Unternehmen eine Ausweichtaktik benutzt. Kein Feedback mehr an Bewerber/ innen oder nur noch in Ausnahmefällen bei eigenen Auszubildenden, lautet die Devise in 19 Prozent (10) der befragten Unternehmen. „Wir schreiben so einen lapidaren Satz, der ist mit den Anwälten abgestimmt. “ (Geschäftsführer, Großunternehmen, priv. Dienstleistungen). Diese Reduktion der Kommunikation mit erfolglosen Bewerber/innen wird von vielen Personalverantwortlichen ausdrücklich bedauert, weil sie diesen Lernchancen verbaue.

Bei der Personalentwicklung hat das AGG bisher noch nichts bewirkt. Niemand benennt irgendwelche Veränderungen. Auch auf Kündigungen hat das AGG bisher keine Auswirkungen gehabt. 90 Prozent der Befragten verneinen hier jede Veränderung.

\section{Entgeltpolitik}

Frauen sind beim Entgelt gerade in Deutschland im europäischen Vergleich besonders benachteiligt. Der Bruttostundenverdienst von Frauen lag nach den Ergebnissen der Verdienststrukturerhebung des Statistischen Bundesamtes 2007 um 23 Prozent unter dem der Männer. ${ }^{10}$ Allerdings ist nur ein Teil dieser Entgeltdifferenz auf direkte oder mittelbare Diskrimi-

10 Vgl. Stat. Bundesamt: Verdienstunterschied zwischen Männern und Frauen im Westen höher als im Osten, Pressemitteilung Nr. 427 vom 14.11.2008. 
nierung wegen des Geschlechts zurückzuführen. Hatte die Vorläuferregelung zum AGG in $\$ 612$ Abs. 3 BGB Entgeltdiskriminierung noch ausdrücklich verboten, wird Entgelt in $\mathbb{\int} 8$ Abs. 2 AGG nur noch im Zusammenhang mit Ausnahmen vom allgemeinen Diskriminierungsverbot des $\$ 7$ AGG angesprochen und damit gesetzlich wieder unsichtbar gemacht. Diese Verschlechterung dürfte kaum dazu beitragen, Unternehmen für Entgeltbenachteiligung im eigenen Bereich zu sensibilisieren.

Das zeigte auch die Befragung. Nur zwei Befragte benannten Veränderungen im Entgeltbereich. In einem Großunternehmen der Elektrobranche erhalten behinderte Mitarbeiter in unteren Lohngruppen seit 2007 einen neuen Bonus von 50 Euro im Monat. Einmal gab es eine Angleichung nach unten durch Streichung bisheriger Privilegien. 87 Prozent (45) der Befragten verneinten jede Veränderung. In einem Kleinunternehmen aus dem Bereich der Konsumgüterproduktion war zwar bei einer Umfrage herausgekommen, dass sich die Frauen bei der Entlohnung gegenüber den Männern ein bisschen benachteiligt fühlten. Doch war das kein Anlass, das bestehende Entgeltsystem zumindest transparenter zu gestalten. Es bleibt abzuwarten, ob die Frauen im äußersten Fall jetzt gegen solche Entgeltbenachteiligung aufgrund des AGG klagen oder sich an die neu geschaffene Antidiskriminierungsstelle des Bundes wenden werden.

Tarifverträge wurden nur zwei Mal im Zusammenhang mit dem neuen Entgeltrahmentarifvertrag (ERA) des Metallbereichs problematisiert. Die betrieblichen Akteure haben die kollektivvertragliche Ebene bei der Frage nach Entgeltdiskriminierung wenig im Blick und können vermutlich tarifvertragliche Details auch nur schlecht auf Diskriminierung hin beurteilen. Dass das AGG über $\mathbb{S} 15$ Abs. 3 Unternehmen von der Haftung für Diskriminierungen durch Kollektivverträge weitgehend freistellt, dürfte das Ausblenden der Kollektivebene bei der Suche nach Diskriminierung zusätzlich begünstigen.

\section{Akzeptanz von religiösen Zeichen}

Interessant sind die Einstellungen der betrieblichen Akteurinnen und Akteure zu religiösen und weltanschaulichen Zeichen. Deshalb fragten wir beispielhaft, ob muslimische Frauen mit Kopftuch im Betrieb akzeptiert würden. Daraufhin ergaben sich große Unterschiede je nach persönlichen Einstellungen oder Werten in der Unternehmenskultur, die in drei Gruppen eingeteilt werden können:

Etwa ein Viertel der Befragten duldet das Kopftuch als religiöses Zeichen nicht, und zwar mit den Begründungen, dass im Betrieb Neutralität gefordert werde, ein Kopftuch genauso unerwünscht wie Piercing oder Plaketten sei und dass es nicht zur Unternehmenskultur passe: „Als wir eine Stelle in der Buchhaltung ausgeschrieben haben, da hatten wir 80 Bewerbungen, da waren auch 20 Kopftücher dabei, die wurden gleich aussortiert.“, drückt es unverblümt der Personalleiter eines Großunternehmens aus und gibt als Begründung „die Störung des Betriebsfriedens“ an. Bei den dezidiert ablehnenden Haltungen überwiegen allerdings die kleineren Betriebe.
Knapp die Hälfte der Befragten hat explizit keine Probleme mit kopftuchtragenden Beschäftigten und gibt als Begründung an: gehört zur Kundenorientierung, war schon immer zugelassen, darauf wird nicht geschaut. „Da habe ich gar kein Problem mit. Es wäre bestimmt komisch, wenn hier jemand mit einem Kopftuch rumlaufen würde. Aber ich glaube, man würde erstmal gucken und dann wäre es okay. “ (Personalleiterin, Großunternehmen, Maschinenbau).

Das restliche Viertel der Betriebe differenziert bei eingeschränkter Toleranz. Die Befragten drücken keine generell ablehnende Haltung aus, sind aber Kopftuch tragenden Mitarbeiterinnen gegenüber nicht uneingeschränkt positiv eingestellt. Die Tatsache, dass viele mit dem Problem noch nichts zu tun hatten, - „Diese Situation hatten wir zum Glück noch nicht. “ - wird begrüßt. Zumeist wird nach Arbeitsbereich unterschieden: Beschäftigte ohne Kundenkontakt, vor allem im Reinigungsdienst oder im Küchenbereich, dürfen Kopftuch tragen, aber „kein Kopftuch am Empfang.“ (Geschäftsführerin, KUM, Personaldienstleistungen). „Hinten (in der Küche, d. Verf.) haben wir damit keine Probleme, vorne (im Restaurant, d. Verf.) geht das gar nicht." (Geschäftsführer, KMU, Gastronomie). „Kopftuch? ... Im Außendienst würde das wohl nichts werden.“ (Betriebsrat, KMU, Konsumgüter).

Die Kopftuch-Diskussion vor den Gerichten zentrierte sich bislang auf den öffentlichen Dienst, insbesondere Lehrerinnen, und hat Unternehmen der Privatwirtschaft bislang kaum erreicht. Dort jedoch ist jede Ablehnung einer Bewerbung aufgrund eines weltanschaulichen Zeichens ein Verstoß gegen $\ 7$ Abs. 1 AGG, wenn nicht eine bestimmte Religion eine nach Art der Tätigkeit gerechtfertigte berufliche Anforderung darstellt. Darunter fällt die sog. „Kirchenklausel“ des $\mathbb{9}$ Abs. 1 AGG. Doch selbst in der christlichen Kirche dürfen nichtchristliche Bewerber/innen nicht generell abgelehnt werden, sondern sind im sog. verkündungsfernen Bereich zuzulassen. ${ }^{11}$ Die Ablehnung einer kopftuchtragenden Bewerberin aufgrund einer Tätigkeit mit Kundenkontakt oder im Service würde im privatwirtschaftlichen Bereich von den Gerichten nicht akzeptiert werden. ${ }^{12}$ Bislang gibt es hier jedoch kaum Konfliktfälle, wohl auch weil bis jetzt die meisten Beschäftigten mit Kopftuch als Reinigungskräfte, Küchenhilfen und dergleichen arbeiten. Dies könnte sich aber in Zukunft ändern, wenn sich kopftuchtragende Hochschulabsolventinnen - und solche gibt es zunehmend - auf qualifizierte Arbeitsplätze bewerben und selbstbewusst ihre Rechte fordern. Die Betriebe müssen hier dazulernen und ihre Prinzipien überdenken, nicht nur um Konflikte mit dem Gesetz zu vermeiden, sondern auch um qualifizierte Arbeitskräfte nicht aufgrund persönlicher Vorurteile zu verlieren.

Drei Unternehmen haben Gebetsräume oder gestatten Gebetszeiten, die dann nachgearbeitet werden müssen. Zwei Befragte aus Kleinunternehmen lehnten Gebetszeiten ausdrück-

\section{Vgl. Bauer; Göpfert; Krieger § 9 Rn 15.}

12 Das BAG hat schon 2002 im Verkauf pro Kopftuch entschieden und befürchtete Kundenvorbehalte nicht als Rechtfertigung akzeptiert: BAG 10.10.2002 AP Nr. 44 zu § 1 KSchG 1969; bestätigt durch BVerfG (Kammer) v. 30.7.2003 Az. 1 BvR 792/03. 
lich ab. Immerhin wird hier bezogen auf Beschäftigte mit islamischem Hintergrund eine vorsichtige Öffnung der Betriebe erkennbar. Tatsächlich hat das Landesarbeitsgericht Hamm ${ }^{13}$ bereits 2002 entschieden, dass ein gläubiger Arbeitnehmer wegen seines Grundrechts auf Glaubensfreiheit aus Art. 4 Abs. 1 und 2 GG berechtigt sein kann, seinen Arbeitsplatz zur Verrichtung kurzer Gebete zu verlassen, solange betriebliche Störungen durch zumutbare Änderungen der Organisation vermieden werden können. Das AGG bringt in diesem Punkt also keine neuen Arbeitgeberverpflichtungen, macht aber bereits bestehende vielleicht bewusster.

\section{Positive Maßnahmen}

71 Prozent (37) der Befragten verneinten generell eine gezielte Gleichstellungspolitik des Unternehmens: „Personalpolitik wird aus dem Bauch entschieden.“ „Fachliches steht im Vordergrund.“ Eingestellt werde, wer „zur Firma passt“. Drei Mal wurde darauf verwiesen, dass eine Gleichstellung von Frauen im Betrieb bereits gegeben sei oder Frauen schon heute die Mehrzahl der im Betrieb Beschäftigten stellten. Andere verwiesen darauf, dass Einzelfallförderung sehr wohl betrieben werde, man das aber im Gegensatz zu anderen Unternehmen nicht an die große Glocke hänge. Zwei Mal wurde berichtet, dass bestehende Förderungsinstrumente - Gleichstellungsbeauftragte bzw. Betriebskindergarten - wieder abgeschafft worden seien, weil sie sich als nicht erforderlich oder zu teuer erwiesen hätten.

Eine Minderheit von 35 Prozent (18) der Befragten gab an, positive Maßnahmen zur Förderung bestimmter Beschäftigtengruppen im Unternehmen zu haben. Solche Maßnahmen waren: Kindergartenplätze, Betriebskindergärten oder ElternKind-Zimmer im Betrieb, weiche Frauenquoten bei Kündigungen, Väterprojekte, Projekt Managerinnen 50+ und Geschlechtergerechtigkeit bei Einstellungen ohne Quotierung, Aktionen „Frauen in technische Berufe“ oder Seminare für ältere Mitarbeiter. Einzelne Unternehmen, zumeist große, zeigen hier viel Engagement und Erfindungsgeist.

Doch nur zwei Befragte meinten, dass eine positive Maßnahme wegen des AGG neu eingeführt worden sei, nämlich der Zusatz in Anzeigen, bei gleicher Qualifikation würden Frauen und/oder Schwerbehinderte bevorzugt. Diejenigen, die jeden Zusammenhang mit dem AGG ablehnten, argumentierten zum Beispiel: „Wir hatten schon vorher alles.“ oder „Keine Diskriminierung der Männer durch Frauenbeauftragte erwünscht. “ Die Ansicht der meisten Befragten dürfte sich aber in dem bloßen Hinweis widerspiegeln: „Nein, da machen wir gar nichts."

Immerhin 19 Prozent (10) der Befragten meinen, in ihren Unternehmen werde die Gleichstellung der Geschlechter aktiv verfolgt, drei Unternehmen sind sogar einschlägig zertifiziert. Großbetriebe überwiegen. In zwei weiteren Unternehmen hätte man gern Männerquoten, ohne allerdings geeignete Männer finden zu können. Gender Mainstreaming oder Managing Diversity spielten in den befragten Unternehmen noch keine wirkliche Rolle.
Sogar 27 Prozent (14) der Befragten verweisen auf die besondere Familienfreundlichkeit ihrer Unternehmenspolitik, zwei Mal im Zusammenhang mit dem Audit Beruf und Familie. 65 Prozent (34) verfolgten auch keine besondere Personalpolitik zugunsten von Familien. Manche hielten eine Vereinbarkeit von Familie und Beruf ausdrücklich für schwierig: „Wenn die Kinder krank werden, fliegt uns der Dienstplan weg. “ Nur eine oder wenige Mütter könnten im jeweiligen Kleinbetrieb verkraftet werden. Andere hatten keine wirkliche Vorstellung, was Familienfreundlichkeit bedeuten würde: Eine Personalreferentin hielt schon die Planung regelmäßiger Familienfeste für Betriebsangehörige für familienfreundlich. Einem Betriebsrat und AGG-Beauftragten aus dem Metallbereich fiel zu familienfreundlicher Personalpolitik nur der freie Sonnabend ein getreu der alten IGM-Forderung „Sonnabends gehört Vati mir“. Die Personalverantwortliche eines Großbetriebes meinte, eine Frau müsse sich eben zwischen Kind und Beruf entscheiden: „Wenn ich so einen kleinen Wurm habe, dann muss ich damit leben, dass ich zwei oder drei Jahre nicht zur Arbeit gehe.“ Der Geschäftsführer eines Kleinbetriebes schließlich hielt Elternförderung für eine Diskriminierung Kinderloser.

17 Prozent (9) der Befragten äußerten deutliche Vorbehalte gegenüber positiven Maßnahmen, insbesondere Frauenquoten: „Von Frauenquoten halte ich gar nichts.“ „Ich möchte keine Quotenfrauen! Und auch keine Quotenmänner!“ „Die guten Mitarbeiter landen gar nicht auf Ihrem Tisch, weil Sie so sehr mit Ihren guten Quoten beschäftigt sind!“ Manche sahen Fördermaßnahmen als Diskriminierung der Geförderten an oder hatten sich selbst als angebliche „Quotenfrau“ sogar schon diskriminiert gefühlt. Andere wussten nichts von der Ausnahmeregelung des $\ 5$ AGG: „Wenn ich etwas für Muttis mache, ist das nicht diskriminierend den Kinderlosen gegenüber? “

Generell lässt sich feststellen, dass das AGG keinen Anreiz darstellt, Fördermaßnahmen über die rechtlichen Verpflichtungen hinaus einzuführen, bestehende aber auch nicht gefährdet. Die - wenigen - Unternehmen, die schon immer aktiv in Gleichstellungspolitik waren, sind es weiterhin, während die - zahlreicheren - Unternehmen, die in dieser Hinsicht abstinent sind, dies auch bleiben.

\section{Benachteiligung bestimmter Beschäftigtengruppen dauert an}

Die Abstinenz so vieler Unternehmen im Bereich aktiver Gleichstellungspolitik und die geringen Veränderungen im Diskriminierungsschutz wären unbedenklich, wenn in den Unternehmen tatsächlich keine Benachteiligungen mehr existieren würden. Die Befragten wollten hier, nach ihrer persönlichen Meinung befragt, jedoch überwiegend keine Entwarnung geben. Zwei Drittel (35) der Befragten sahen sehr klar umrissene Benachteiligungen. Nur ein Drittel (17) meinte, in ihren Unternehmen werde nicht benachteiligt. Bei vier Unternehmen wurden gleich drei bis fünf Merkmalsgruppen als benachteiligt eingestuft, quasi Hardliner.

13 LAG Hamm 26.2.2002, AP Nr. 3 zu § 611 BGB Gewissensfreiheit. 
An der Spitze lag die Frauenbenachteiligung mit 22 Nennungen. Mit 17 Nennungen folgte Benachteiligung wegen der Religion. Noch elf sahen in ihren Unternehmen Altersbenachteiligungen, zumeist, aber nicht immer bei Älteren. Nur einzelne jedoch sahen in ihren Betrieben Behinderte oder Homosexuelle benachteiligt. Andererseits hatten anfangs 20 Befragte erklärt, ihr Unternehmen erfülle die Schwerbehindertenquote nicht, und sieben sagten, es würden überhaupt keine Menschen mit Behinderung im Betrieb beschäftigt. Nur acht Befragte wussten um irgendwelche Homosexuelle im eigenen Betrieb. Hier dürfte es also wohl weniger an Benachteiligungen als an der Wahrnehmung von Benachteiligung fehlen. Viele Befragte halten das AGG im eigenen Unternehmen für unnötig, da ohnehin nicht diskriminiert würde, in anderen Unternehmen aber für notwendig.

\section{Konflikte bei der Umsetzung des AGG}

Die Klageflut ist ausgeblieben. Und unternehmensintern hat es ebenfalls nur wenige Konflikte gegeben. Nur 17 Prozent der Befragten berichteten von Konfliktfällen, 75 Prozent verneinten diese Frage ausdrücklich. Zwei Befragte führten diese Ruhe darauf zurück, dass von Benachteiligung Betroffene so schlecht informiert seien: „Die meisten wissen gar nicht, was im AGG drin steht. Deswegen hatten wir nämlich auch noch keine Klagen.“ Auf eine geschlechtsspezifisch formulierte Anzeige „Sekretärin“ habe es 100 Bewerbungen gegeben, aber niemand hat sich beschwert. Vorhandene Konflikte wurden intern gelöst, nur einmal wurde von einer Klage berichtet. Sogenanntes AGG-Hopping wurde zwei Mal beobachtet.

\section{Aufwand und Kosten}

Unsere Studie liefert keine Anhaltspunkte dafür, dass die Umsetzung des AGG den Unternehmen erhebliche Kosten verursacht hätte. Mehr Personal hat das AGG nur aus Sicht zweier Befragter gefordert. 90 Prozent der Befragten meinen, dass die Arbeit mit demselben Personal wie vorher erledigt werde. Beim Zeitaufwand waren die Meinungen gespalten: 44 Prozent sahen keinen höheren Zeitaufwand, 54 Prozent bejahten ihn. Die meisten Befragten begründeten den zusätzlichen Zeitaufwand jedoch durch einmalige Einführungsmaßnahmen und Schulungen. Nur wenige meinten, dass generell mehr Zeit für Auswahlgespräche oder die Dokumentation verwendet werde. Insgesamt betrachtet hat das AGG den Zeitaufwand somit vor allem einmalig, nämlich bei seiner Einführung steigen lassen. Das bestätigen auch die Antworten auf die direkte Frage nach den Kosten, die überwiegend als einmalig klassifiziert werden. Ansonsten sind die Antworten auch hier gespalten: Keine Kostensteigerung sahen 48 Prozent der Befragten, 48 Prozent sahen im Gegenteil Kostensteigerungen.

Abschließend erklärten viele der Befragten in diesem $\mathrm{Zu}$ sammenhang, das AGG sei überschätzt worden, das meiste habe man schon gelebt. „Das AGG hat bei uns intern absolut keine Rolle gespielt.“ Oder wegen der Kosten und des geringen Risikos bei Gesetzesbrüchen setzten die Betriebe manches einfach nicht um. Nur drei Befragte hielten das AGG samt seinem Aufwand für problematisch.

Vor allem die Anwälte und Berater hätte am Gesetz verdienen wollen: „Wenn die Anwälte uns nicht so vollgemüllt hätten, hätten wir gar nicht gemerkt, dass das Gesetz eingeführt wurde!“ „Die (Rechtsanwälte und Personalberater, d. Verf.) haben richtig Druck gemacht, weil die natürlich Aufträge haben wollten ... absoluter Wahnsinn!“ Man möchte da fast sagen, dass die wahren AGG-Hopper nicht Scheinbewerber/ innen, sondern diese Angstmacher waren. Offenbar hat es jedoch nicht funktioniert. Nur selten haben Unternehmen in Folge dieser Angst-Kampagne nämlich auch tatsächlich externen fachlichen Rat und externe Schulungsangebote von Anwalts- und Beratungsfirmen außerhalb ihrer Unternehmensverbände in Anspruch genommen.

\section{Beurteilung des AGG durch die Befragten}

Nachdem das AGG zuvor im Detail als wenig wirksam dargestellt worden war, muss die verhalten positive Beurteilung des Gesetzes bei fast der Hälfte der Befragten ganz am Ende der Befragung doch etwas überraschen. 46 Prozent (24) der Befragten beurteilten das Gesetz persönlich positiv. Die Idee, die Zielsetzung und die Tatsache, dass es überhaupt so ein Gesetz gebe, wurden gelobt. Das AGG werde gebraucht und sei wegen der Benachteiligungen in vielen Bereichen sinnvoll. Allerdings dauere es „bestimmt noch Jahre, bis das in den Köpfen verankert ist".

Eine negative Bilanz des Gesetzes zogen nur 37 Prozent (19) der Befragten, etwa zur Hälfte aus KMU und zur Hälfte aus großen Unternehmen, bis auf zwei Beauftragte sämtlich Mitglieder der Geschäftsleitung oder des Personalbereichs. Das AGG sei ein „typisch deutsches Gesetz“ und „völlig überzogen“, „unsinnig“ und nicht der richtige Weg. Keiner wisse mehr, was eigentlich gesucht werde. „Die, die diskriminieren, tun es weiterhin. Ihre Argumente legen sie sich zurecht.“ Eine Mehrheit von 52 Prozent (27) der Befragten meinte allerdings, das AGG habe, egal ob gut oder schlecht, zumindest im eigenen Unternehmen gar keinen Einfluss gehabt.

\section{Fazit}

Die befürchtete Klageflut hat es nicht gegeben. Auch intern hat es offenkundig keine Konfliktwelle in den Betrieben gegeben. Hohe Kosten für die Betriebe hat das neue AGG weder bei seiner Implementierung noch dauerhaft verursacht. Die Personalpolitik deutscher Betriebe dümpelt auf dem bisherigen, zumeist gleichstellungspolitisch wenig innovativen $\mathrm{Ni}$ veau vor sich hin. Nur eine Minderheit von Betrieben ist über die Vorgaben des AGG hinaus gleichstellungspolitisch aktiv, obwohl es offenbar noch viel Benachteiligung in der Privatwirtschaft gibt, vor allem bei Frauen, Älteren und Migranten.

Das Gesetz ist eine notwendige, aber keine hinreichende Bedingung für den Abbau von Benachteiligungen. „Wenn man etwas nicht will, dann macht man es auch nicht! (Personalleiter, Großunternehmen, Maschinenbau). Möglicherweise war die „Angstmache“ im Vorfeld ein gutes Mittel, die Unter- 
nehmen zur Auseinandersetzung mit dem AGG zu motivieren, da ansonsten die Gesetzesänderung völlig ignoriert worden wäre. Bessere Beweisregelungen und schärfere Sanktionen könnten allerdings den Druck zur Umsetzung verstärken. So betrachtet ist die eingetretene Entspannung auch kritisch zu sehen: Offenbar wird wenig Veränderungsdruck erlebt. Manche brachten in der Befragung beiläufig zum Ausdruck, dass in den ausländischen Müttern oder Töchtern ihrer Unternehmen gleichstellungspolitisch mehr gemacht werde, - weil der gesetzliche Druck hier stärker sei und/oder das Bewusstsein ein anderes.

Die größte Wirkung erzielt das AGG bislang also symbolisch: Es drückt den politischen Willen zur Gleichstellung ebenso wie eine gesellschaftliche Sensibilität gegenüber Diskriminierungen aus. Politische und soziale Werte aber können von Betrieben dauerhaft nicht gänzlich ignoriert werden. Nichtdiskriminierung und Gleichstellung bisher benachteiligter Beschäftigtengruppen sind dabei eigentlich nicht nur ein Gebot sozialer Gerechtigkeit sowie gemeinschaftsrechtlicher Verpflichtungen der Bundesrepublik Deutschland. Sie wären auch ein Gebot wirtschaftlicher Vernunft und zukunftsweisender Personalpolitik, sich künftig neue Personengruppen auf einem Arbeitsmarkt zu erschließen, der demografisch bedingt schrumpft und immer „älter“ wird. Das neue AGG böte die Chance, den deswegen in den Betrieben erforderlichen personalpolitischen Wandel in geordneten Bahnen und weitgehend im Gleichschritt aller Unternehmen, also ohne innerstaatliche oder europäische Konkurrenz einzuleiten. Diese Chance wird in den Unternehmen bislang jedoch zu selten gesehen und noch seltener genutzt.

\section{Rezension: Justitia ist eine Frau}

Barbara Degen: Justitia ist eine Frau. Geschichte und Symbolik der Gerechtigkeit. Katalog zu der Ausstellung „Füllhorn, Waage, Schwert - Justitia ist eine Frau“". Opladen und Farmington Hills 2008

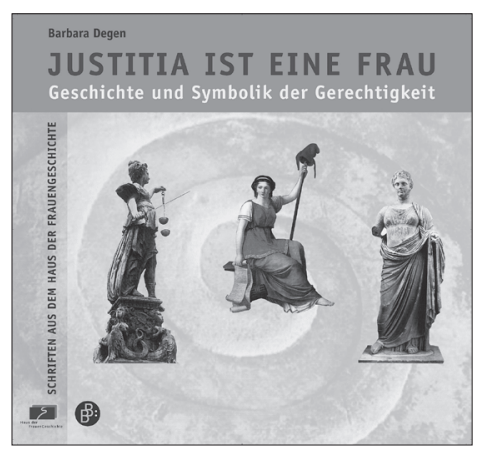

Jeder Juristin ist natürlich geläufig, dass Justitia, die Dame mit der Waage und dem Schwert, als Sinnbild der Gerechtigkeit fungiert. Dieses Symbol ist uns vertraut und es wirkt selbstverständlich. Stellen Sie sich dagegen einen wehrhaften Krieger in der gleichen Funktion vor, lassen Sie sich etwas Zeit: dass er ein Schwert trägt, ist selbstverständlich, aber was will er mit der Waage? Sehen Sie einen weisen alten Mann mit der Waage? Ja, die passt zu ihm, aber das Schwert? Das dürfte ihn überfordern.

Derartige Konnotationen haben wir nicht, wenn wir an die besagte Dame denken, ihre Attribute: Ausgewogenheit, Wehrhaftigkeit und Unvoreingenommenheit erscheinen als notwendige Voraussetzungen einer gerechten Ordnung und es ist keine Frage, dass diese vereint sein müssen, um gute Ergebnisse zu erzielen und: dass Frauen hierzu über die erforderlichen Fertigkeiten verfügen. Jedenfalls erscheint uns die Zuordnung des Symbolgehaltes zu dieser speziellen weiblichen Gestalt folgerichtig. Aber warum?

Barbara Degen ist seit vielen Jahren dieser Frage auf der Spur. Dabei hatte sie eine „einfache Ausgangsthese: Gerechtigkeit ist im Kern Mütterlichkeit, Weisheit und Liebe, und zwar für Kinder, Frauen und Männer“ (S. 12). Sie hat die Geschichte des Symbols verfolgt und ist dabei unvermeidlich auch auf die Geschichte unserer Suche nach der Gerechtigkeit gestoßen. Sie hat herausgefunden, dass es seit jeher offenbar vorrangig, teil- weise ausschließlich, Sache der Frauen war, für eine gerechte Verteilung zu sorgen, was man sich auch zwanglos vorstellen kann: Frauen mussten ihre Kinder ernähren und wenn ihre eigenen Mittel hierfür nicht mehr ausreichten, als die Kinder heranwuchsen, dafür sorgen, dass die Männer von der Jagdbeute etwas abgaben, ohne sich übervorteilt zu fühlen. Höhlenzeichnungen und frühzeitliche Plastiken weisen auf diese Zusammenhänge hin. Untrennbar scheinen Gerechtigkeitsvorstellungen allerdings auch mit der Erfahrung von Geburt und der Verantwortung für das neue Leben verbunden gewesen zu sein. Die gesellschaftliche Macht der Frauen entsprang „aus ihrer potenziellen und realen Möglichkeit, Kinder zu gebären und sie für die Welt zu erziehen“. Ihre Weisheit und ihre Erfahrungen waren gefragt. Die griechische, aber auch die ägyptische Mythologie nehmen diese Zusammenhänge auf. Die verschiedenen Göttinnengestalten, die ihnen zugedachten Charaktere, die mit ihnen verbundenen Sagen reflektieren und tradieren das Wissen über diese weiblichen Fähigkeiten. Offensichtlich schon sehr früh gab es Bemühungen, die eigene körperliche weibliche Erfahrung in größere Zusammenhänge zu stellen. Schon sehr früh waren Frauen einer „natürlichen Ordnung“ des Lebens auf der Spur. Überlegungen zu den Prinzipien eines höheren Rechts, die Frieden und ein gedeihliches Miteinander in der Gemeinschaft sichern, Prinzipien, denen es sich zu nähern gilt, wurden offenbar schon vor vielen Jahrtausenden angestellt. Degen belegt dies durch Bilder und originale Texte, die zeigen, dass die Suche nach Gerechtigkeit eine ganz universelle menschliche Aufgabe ist und dass Frauen zentral daran mitwirkten. So gab es in der frauenzentrierten Kultur des alten Ägypten eine hochentwickelte Weltanschauung, die ihr gedankliches Fundament in der Überzeugung fand, „Frauen seien die Urschöpferinnen und Ordnerinnen des Universums“ (S. 27). Ägyptische Pharaonen wurden von ihren Müttern in-

1 S. hierzu bereits djbZ 4/2008, S. 192. 